\title{
MAXIMAL MOMENT INEQUALITIES FOR STOCHASTIC PROCESSES
}

\author{
F. MÓRICZ
}

(Communicated by J. Marshall Ash)

\begin{abstract}
Let $X_{a, b}$ be nonnegative random variables with the property that $X_{a, b} \leq X_{a, c}+X_{c, b}$ for all $0 \leq a<c<b \leq T$, where $T>0$ is fixed. We define $M_{a, b}:=\sup \left\{\bar{X}_{a, c}: a<c \leq b\right\}$ and establish bounds for $E M_{a, b}^{\gamma}$ and $E \exp \left(\lambda M_{a, b}\right)$ in terms of assumed bounds for $E X_{a, b}^{\gamma}$ and $E \exp \left(\lambda X_{a, b}\right)$, respectively, where $\gamma \geq 1$ and $\lambda$ runs through an interval $\left(\lambda_{0}, \infty\right)$ with fixed $\lambda_{0} \geq 0$. These bounds explicitly involve a nonnegative function $g(a, b)$ assumed to be quasi-superadditive with an index $Q$, which means that $g(a, c)+g(c, b) \leq Q g(a, b)$ for all $0 \leq a<c<b \leq T$, where $1 \leq Q<2$ is fixed.

Maximal inequalities obtained in this way can be applied to stochastic processes exhibiting long-range dependence. Among others, these applications may include certain self-similar processes such as fractional Brownian motion, stochastic processes occurring in linear time-series models, etc.
\end{abstract}

\section{INTRODUCTION}

Let $X_{a, b}=X_{a, b}(\omega)$ be nonnegative random variables (in abbreviation: r.v.'s) defined for all $0 \leq a<b \leq T$ and $\omega \in \Omega$, in such a way that

$$
X_{a, b} \leq X_{a, c}+X_{c, b} \text { for all } 0 \leq a<c<b \leq T \text {. }
$$

Here $T>0$ is a fixed real number, $\Omega$ a probability space, and (1.1) expresses a subadditive property of the variables $X_{a, b}$.

For instance, if $Y_{t}$ is a real- or complex-valued stochastic process defined for $0 \leq t \leq T$ and

$$
X_{a, b}:=\left|\int_{a}^{b} Y_{t} d t\right|
$$

then (1.1) is clearly satisfied.

Received by the editors July 6, 1992.

1991 Mathematics Subject Classification. Primary 60G99.

Key words and phrases. Stochastic process, maximal fluctuation, quasi-superadditivity, moment inequality, power type estimate, exponential estimate, continuous time analogue of the RademacherMen'shov inequality.

The author was partially supported by the Hungarian National Foundation for Scientific Research under Grant \#234. 
Remark 1. If we assume that for some $\gamma \geq 1$

$$
\int_{0}^{T}\left(E\left|Y_{t}\right|^{\gamma}\right)^{1 / \gamma} d t<\infty
$$

then we can conclude

$$
E X_{a, b}^{\gamma}<\infty \text { for all } 0 \leq a<b \leq T .
$$

In fact, by the generalized Minkowski inequality,

$$
\begin{aligned}
\left(E X_{a, b}^{\gamma}\right)^{1 / \gamma} & =\left(\int_{\Omega}\left|\int_{a}^{b} Y_{t}(\omega) d t\right|^{\gamma} d \omega\right)^{1 / \gamma} \\
& \leq \int_{a}^{b}\left(\int_{\Omega}\left|Y_{t}(\omega)\right|^{\gamma} d \omega\right)^{1 / \gamma} d t=\int_{a}^{b}\left(E\left|Y_{t}\right|^{\gamma}\right)^{1 / \gamma} d t .
\end{aligned}
$$

Unfortunately, the inequality

$$
\ln \left(\int_{\Omega} \exp \left(\left|\int_{a}^{b} Y_{t}(\omega) d t\right|\right) d \omega\right) \leq \int_{a}^{b} \ln \left(\int_{\Omega} \exp \left(\left|Y_{t}(\omega)\right|\right) d \omega\right) d t,
$$

analogous to $(1.5)$ when the power function $\phi(\lambda):=\lambda^{\gamma}$ is replaced by the exponential function $\phi(\lambda):=e^{\lambda}$, fails to hold in general. In connection with this, we raise the following

Problem 1. Find a condition in terms of $E \exp \left(\lambda\left|Y_{t}\right|\right)$ that guarantees

$$
\left.E \exp \left(\lambda X_{a, b}\right)<\infty \quad \text { (for some or all } \lambda>0\right)
$$

in the same sense as (1.3) implies (1.4).

Define the maximal fluctuation to be

$$
M_{a, b}:=\sup _{a<c \leq b} X_{a, c} \text { for all } 0 \leq a<b \leq T .
$$

We will establish bounds for the quantities $E M_{a, b}^{\gamma}$ and $E \exp \left(\lambda M_{a, b}\right)$ in terms of the assumed bounds for the quantities $E X_{a, b}^{\gamma}$ and $E \exp \left(\lambda X_{a, b}\right)$ for all $0 \leq a<b \leq T$, respectively, where $\gamma \geq 1$ is a fixed real number and $\lambda$ runs through some interval $\left(\lambda_{0}, \infty\right)$ with fixed $\lambda_{0} \geq 0$.

These bounds will relate to the variables $X_{a, b}$ in specified ways through some function $g(a, b)$ possessing the following properties:

(i) $g(a, b) \geq 0$ for all $0 \leq a<b \leq T$,

(ii) $g(a, b)$ is continuous in $b$ for fixed $a$,

(iii) $g(a, b)$ is nondecreasing in $b$ for fixed $a$,

(iv) $g(a, c)+g(c, b) \leq Q g(a, b)$ for all $0 \leq a<c<b \leq T$,

where $1 \leq Q<2$ is fixed. We call property (1.6)(iv) quasi-superadditivity with the index $Q$ (or simply, $Q$-superadditivity). The case $Q=1$ corresponds to the usual notion of superadditivity. If, instead of (1.6)(iv), we assume

$$
g(a, c)+g(c, b)=g(a, b) \text { for all } 0 \leq a<c<b \leq T,
$$

which means additivity, then (1.6)(iii) follows from (1.6)(i) and (1.7). 
The notion of $Q$-superadditivity was introduced in [7], where various examples were presented. We refer briefly to the fact that when $g(a, b)$ is given by $\operatorname{Var} X_{a, b}$, the case of $Q$-superadditivity for $Q>1$ corresponds, roughly speaking, to the property that $\operatorname{Var} X_{a, b}$ increases slower than $O(b-a)$ as $b-a \rightarrow \infty$. See [9] for examples including fractional Brownian motion and other self-similar stochastic processes. To mention another scope of applications, the maximal inequalities below can serve as important tools in solving certain problems in system identification, while studying linear time-series models. We refer to [2, $3,4,8]$ in this respect.

We raise the following

Problem 2. Conditions (i), (iii), and (iv) in (1.6) seen to be natural, while (ii) seems to be of technical character. It certainly makes the proofs of Theorems 1-6 below very convenient. But we think condition (1.6)(ii) may be deleted or at least weakened for the left (or right) continuity of $g(a, b)$ in $b$ for fixed $a$, without affecting the validity of Theorems $1-6$. We are unable to drop this continuity condition in our proofs.

\section{Power type estimates: CASE $\alpha>1$}

We assume that there exists a number $\gamma \geq 1$ such that condition (1.4) is satisfied.

Theorem 1. Let $\alpha>1,1 \leq Q<2^{(\alpha-1) / \alpha}, \gamma \geq 1$, and $T>0$ be given numbers. Suppose that there exists a function $g(a, b)$ satisfying the conditions in (1.6) such that

$$
E X_{a, b}^{\gamma} \leq g^{\alpha}(a, b) \text { for all } 0 \leq a<b \leq T
$$

and

$$
M_{T}:=\sup _{0 \leq a<b \leq T} \frac{E M_{a, b}^{\gamma}}{g^{\alpha}(a, b)}<\infty
$$

Then

$$
E M_{a, b}^{\gamma} \leq Q^{\alpha}\left(2^{\alpha / \gamma}-2^{1 / \gamma} Q^{\alpha / \gamma}\right)^{-\gamma} g^{\alpha}(a, b) \text { for all } 0 \leq a<b \leq T .
$$

We note that Gerencsér [2] studied the particular case when

$$
Y_{t}:=f_{t} u_{t}, \quad g(a, b):=\int_{a}^{b} f_{t}^{2} d t, \quad \text { and } \quad \alpha=\gamma / 2
$$

in (1.2), where $f_{t}$ is a square integrable function over the interval $[0, T], u_{t}$ is an $L$-mixing process subject to certain further assumptions, and $\gamma>2$. In this case, condition (2.2) is clearly satisfied since

$$
M_{T} \leq T^{1 / 2} \sup _{0 \leq t \leq T}\left(E\left|u_{t}\right|^{\gamma}\right)^{1 / \gamma}
$$

(see the proof of [2, Theorem 5.1]). We note that the constant received in [2] is different from that in (2.3).

Proof of Theorem 1. It is a continuous time modification of the method applied in [5] for sequences of r.v.'s. 
If $g(a, b)=0$ for some $0 \leq a<b \leq T$, then by (1.6)

$$
g(a, c)=0 \text { for all } a<c \leq b .
$$

By (2.1), $X_{a, c}=0$ a.s. Consequently, $M_{a, b}=0$ a.s. and (2.3) is trivially satisfied in this case.

From now on, assume that $g(a, b) \neq 0$. By (1.6), choose a number $c$ such that $a<c<b$ and

$$
g(a, c)=\frac{Q}{2} g(a, b) .
$$

Hence

$$
g(c, b) \leq Q g(a, b)-g(a, c)=\frac{Q}{2} g(a, b) .
$$

It is plain that

$$
M_{a, b} \leq X_{a, c}+\left(M_{a, c}^{\gamma}+M_{c, b}^{\gamma}\right)^{1 / \gamma},
$$

whence, by Minkowski's inequality,

$$
\left(E M_{a, b}^{\gamma}\right)^{1 / \gamma} \leq\left(E X_{a, c}^{\gamma}\right)^{1 / \gamma}+\left(E M_{a, c}^{\gamma}+E M_{c, b}^{\gamma}\right)^{1 / \gamma} .
$$

Introduce the quantity

$$
m_{a, b}:=\frac{E M_{a, b}^{\gamma}}{g^{\alpha}(a, b)} \text { for all } 0 \leq a<b \leq T .
$$

Clearly,

$$
M_{T}=\sup _{0 \leq a<b \leq T} m_{a, b}
$$

(cf. (2.2)). Using this notation, from (2.1) and (2.6) it follows that

$$
\left\{g^{\alpha}(a, b) m_{a, b}\right\}^{1 / \gamma} \leq g^{\alpha / \gamma}(a, c)+\left\{g^{\alpha}(a, c) m_{a, c}+g^{\alpha}(c, b) m_{c, b}\right\}^{1 / \gamma} \text {. }
$$

Taking (2.4) and (2.5) into account, we may write

$$
m_{a, b}^{1 / \gamma} \leq\left(\frac{Q}{2}\right)^{\alpha / \gamma}\left\{1+\left(m_{a, c}+m_{c, b}\right)^{1 / \gamma}\right\}
$$

Hence, by (2.7),

$$
m_{a, b}^{1 / \gamma} \leq\left(\frac{Q}{2}\right)^{\alpha / \gamma}\left(1+2^{1 / \gamma} M_{T}^{1 / \gamma}\right) .
$$

Since this inequality holds for all $0 \leq a<b \leq T$, we also have

whence

$$
M_{T}^{1 / \gamma} \leq\left(\frac{Q}{2}\right)^{\alpha / \gamma}\left(1+2^{1 / \gamma} M_{T}^{1 / \gamma}\right)
$$

$$
M_{T} \leq Q^{\alpha}\left(2^{\alpha / \gamma}-2^{1 / \gamma} Q^{\alpha / \gamma}\right)^{-\gamma} .
$$

This is equivalent to (2.3) and the proof is complete.

3. Power tYPe estimates: CASE $\alpha=1$

In this section, we confine our attention to the particular case when $Q=1$ in (1.6). We observe that in this case the constant $\left(2^{\alpha / \gamma}-2^{1 / \gamma}\right)^{-\gamma}$ in (2.3) tends to $\infty$ as $\alpha$ approaches 1 from above.

In the sequel, $\lceil u\rceil$ denotes the upper integral part of the real number $u$, the logarithm is to base 2 , and $\log ^{+} u:=(0, \log u)$. 
Theorem 2. Let $\gamma \geq 1$ and $T>0$ be given numbers. Suppose that there exists a function $g(a, b)$ satisfying conditions (1.6)(i), (iii), (iv) with $Q=1$ such that

$$
E X_{a, b}^{\gamma} \leq g(a, b) \text { for all } 0 \leq a<b \leq T
$$

and

$$
M_{T}:=\sup _{0 \leq a<b \leq T \& b-a \leq 1} \frac{E M_{a, b}^{\gamma}}{g(a, b)}<\infty .
$$

Then

$$
E M_{a, b}^{\gamma} \leq\left(M_{T}^{1 / \gamma}+\left[\log ^{+}(b-a)\right]\right)^{\gamma} g(a, b) \text { for all } 0 \leq a<b \leq T .
$$

Theorem 2 can be considered as the continuous time analogue of the famous Rademacher-Men'shov inequality (see, e.g., [1]).

Proof of Theorem 2. We apply an induction argument with respect to $\lceil b-a\rceil$. If $\lceil b-a\rceil \leq 1$, then (3.3) is an immediate consequence of (3.1) and (3.2).

Now, let $\lceil b-a\rceil \geq 2$. As an induction hypothesis, we assume that (3.3) has been proved for $(a, c)$ and $(b, c)$ instead of $(a, b)$, where $c:=(a+b) / 2$. We repeat the first part of the proof of Theorem 1 and arrive at inequality (2.6). Hence, by (3.1) and the induction hypothesis,

$$
\begin{aligned}
\left(E M_{a, b}^{\gamma}\right)^{1 / \gamma} & \leq g^{1 / \gamma}(a, c)+\left(M_{T}^{1 / \gamma}+\left[\log ^{+}\left(\frac{b-a}{2}\right)\right]\right) g^{1 / \gamma}(a, b) \\
& \leq\left(1+M_{T}^{1 / \gamma}+\left\lceil\log ^{+}\left(\frac{b-a}{2}\right)\right\rceil\right) g^{1 / \gamma}(a, b) \\
& =\left(M_{T}^{1 / \gamma}+\left\lceil\log ^{+}(b-a)\right\rceil\right) g^{1 / \gamma}(a, b) .
\end{aligned}
$$

This is equivalent to (3.3) and the proof is complete.

Remark 2. The case $\alpha=Q=1$ can be treated in a more general setting. To this effect, let $w(\lambda)$ be a positive and nondecreasing function for $\lambda>0$. We introduce another function $W(\lambda)$ by putting

$$
W(\lambda):= \begin{cases}M_{T}^{1 / \gamma} w(\lambda) & \text { for } 0<\lambda \leq 1, \\ w(\lambda / 2)+W(\lambda / 2) & \text { for } \lambda>1,\end{cases}
$$

where $M_{T}$ is defined in (3.6) below.

It is plain that $W(\lambda)$ is also positive and nondecreasing for $\lambda>0$. Furthermore,

$$
W(\lambda)=M_{T}^{1 / \gamma} w\left(\frac{\lambda}{2^{\lceil\log \lambda\rceil}}\right)+\sum_{k=1}^{\lceil\log \lambda\rceil} w\left(\frac{\lambda}{2^{k}}\right) \text { for } \lambda>1 .
$$

Theorem 3. Let $\gamma \geq 1$ and $T>0$ be given numbers. Suppose that there exist a function $g(a, b)$ satisfying conditions (1.6)(i), (iii), (iv) with $Q=1$ and $a$ positive, nondecreasing function $w(\lambda)$ for $\lambda>0$ such that

$$
E X_{a, b}^{\gamma} \leq w^{\gamma}(b-a) g(a, b) \text { for all } 0 \leq a<b \leq T
$$

and

$$
M_{T}:=\sup _{0 \leq a<b \leq T \& b-a \leq 1} \frac{E M_{a, b}^{\gamma}}{w^{\gamma}(b-a) g(a, b)}<\infty .
$$


Then

$$
E M_{a, b}^{\gamma} \leq\left(M_{T}^{1 / \gamma}+W(b-a)\right)^{\gamma} g(a, b) \text { for all } 0 \leq a<b \leq T,
$$

where $W(\lambda)$ is defined in (3.4).

The proof of Theorem 3 is a repetition of that of Theorem 2, with slight modifications. We omit it.

Clearly, Theorem 2 is the particular case of Theorem 3 when $w(\lambda):=1$ for all $\lambda>0$. Another particular case of Theorem 3 reads as follows.

Theorem 4. Let $\beta>0, \gamma \geq 1$, and $T>0$ be given numbers. Suppose that there exists a function $g(a, b)$ satisfying conditions (1.6)(i), (iii), (iv) with $Q=1$ such that

$$
E X_{a, b}^{\gamma} \leq(b-a)^{\beta} g(a, b) \text { for all } 0 \leq a<b \leq T
$$

and

$$
M_{T}:=\sup _{0 \leq a<b \leq T \& b-a \leq 1} \frac{E M_{a, b}^{\gamma}}{(b-a)^{\beta} g(a, b)}<\infty .
$$

Then

$$
E M_{a, b}^{\gamma} \leq\left(M_{T}^{1 / \gamma}+\frac{1}{2^{\beta / \gamma}-1}\right)^{\gamma}(b-a)^{\beta} g(a, b) \text { for all } 0 \leq a<b \leq T \text {. }
$$

In fact, this time $w(\lambda):=\lambda^{\beta / \gamma}$ for $\lambda>0$. By (3.5),

$$
\begin{aligned}
W(\lambda) & :=M_{T}^{1 / \gamma}\left(\frac{\lambda}{2^{k_{0}}}\right)^{\beta / \gamma}+\sum_{k=1}^{k_{0}} w\left(\frac{\lambda}{2^{k}}\right) \\
& <\left(M_{T}^{1 / \gamma}+\frac{1}{2^{\beta / \gamma}-1}\right) \lambda^{\beta / \gamma} \quad \text { for all } \lambda>1,
\end{aligned}
$$

where $k_{0}:=\lceil\log \lambda\rceil$.

\section{EXPONENTIAL ESTIMATES}

Given a number $1 \leq Q<2$, define $p$ and $q$ in such a way that

$$
1<q<\frac{2}{Q} \text { and } \frac{1}{p}+\frac{1}{q}=1 \text {. }
$$

Theorem 5. Let $K>0,1 \leq Q<2, B \geq p Q /(2-q Q), \lambda_{0} \geq 0$, and $T>0$ be given numbers, where $p$ and $q$ are defined in (4.1). Suppose that there exists a function $g(a, b)$ satisfying the conditions in (1.6) such that

(4.2) $E \exp \left(\lambda X_{a, b}\right) \leq K \exp \left(\lambda^{2} g(a, b)\right)$ for all $\lambda>\lambda_{0}$ and $0 \leq a<b \leq T$,

and

$$
M_{T}:=\sup _{\lambda>\lambda_{0}} \sup _{0 \leq a<b \leq T} \frac{E \exp \left(\lambda M_{a, b}\right)}{\exp \left(B \lambda^{2} g(a, b)\right)}<\infty
$$

Then

$$
E \exp \left(\lambda M_{a, b}\right) \leq\left(1+K^{1 / p}\right)^{p} \exp \left(B \lambda^{2} g(a, b)\right)
$$

for all $\lambda>\lambda_{0}$ and $0 \leq a<b \leq T$.

We note that the counterpart of Theorem 5 for sequences of r.v.'s was proved in [6]. 
Problem 3. We conjecture that Theorem 5 holds true with $B$ as close to 1 as we wish, at the expense of increasing the constant $\left(1+K^{1 / p}\right)^{p}$ in (4.4). But we are unable to prove it.

Proof of Theorem 5. Without loss of generality, we may assume again that $g(a, b) \neq 0$ and choose the number $c$ according to (2.4) and (2.5). This time, we start with the obvious inequality

$$
\exp \left(\lambda M_{a, b}\right) \leq \exp \left(\lambda M_{a, c}\right)+\exp \left(\lambda X_{a, c}+\lambda M_{c, b}\right) .
$$

By Hölder's inequality,

$$
\begin{aligned}
E \exp \left(\lambda M_{a, b}\right) \leq & \left\{E \exp \left(q \lambda M_{a, c}\right)\right\}^{1 / q} \\
& +\left\{E \exp \left(p \lambda X_{a, c}\right)\right\}^{1 / p}\left\{E \exp \left(q \lambda M_{c, b}\right)\right\}^{1 / q} .
\end{aligned}
$$

We introduce the quantity

$$
m_{a, b}(\lambda):=\frac{E \exp \left(\lambda M_{a, b}\right)}{\exp \left(B \lambda^{2} g(a, b)\right)}
$$

By (4.3) and (4.6)

$$
M_{T}=\sup _{\lambda>\lambda_{0}} \sup _{0 \leq a<b \leq T} m_{a, b}(\lambda) .
$$

By (4.2), (4.5), and (4.6),

$$
\begin{aligned}
m_{a, b}(\lambda) & \exp \left(B \lambda^{2} g(a, b)\right) \\
\leq & \left\{m_{a, c}(q \lambda)\right\}^{1 / q} \exp \left(B q \lambda^{2} g(a, c)\right) \\
& +K^{1 / p} \exp \left(p \lambda^{2} g(a, c)\right)\left\{m_{c, b}(q \lambda)\right\}^{1 / q} \exp \left(B q \lambda^{2} g(c, b)\right),
\end{aligned}
$$

whence, by (4.3),

$$
\begin{aligned}
m_{a, b}(\lambda) \leq & M_{T}^{1 / q} \exp \left\{B \lambda^{2}(q g(a, c)-g(a, b))\right\} \\
& +K^{1 / p} M_{T}^{1 / q} \exp \left\{\lambda^{2}(p g(a, c)+B q g(c, b)-B g(a, b))\right\} .
\end{aligned}
$$

We take into account that, by (2.4), (2.5), and (4.1),

$$
q g(a, c)-g(a, b)=\left(\frac{q Q}{2}-1\right) g(a, b)<0
$$

and

$$
p g(a, c)+B q g(c, b)-B g(a, b) \leq\left(\frac{p Q}{2}+\frac{B q Q}{2}-B\right) g(a, b) \leq 0 .
$$

Thus, from (4.8) it follows that

$$
m_{a, b}(\lambda) \leq\left(1+K^{1 / p}\right) M_{T}^{1 / q} .
$$

Since this inequality holds for all $\lambda>\lambda_{0}$ and $0 \leq a<b \leq T$, we also have

$$
M_{T} \leq\left(1+K^{1 / p}\right) M_{T}^{1 / q},
$$

which is equivalent to (4.4) and the proof is complete.

Remark 3. The proving method of Theorem 5 enables us to achieve an essentially more general result. To this effect, let $\phi(\lambda)$ be a function defined for $\lambda>\lambda_{0}$ such that $\phi(\lambda)>0$ and for each constant $q>1$ we have

$$
\sup _{\lambda>\lambda_{0}} \frac{\phi(q \lambda)}{\phi(\lambda)}=: \chi(q)<\infty \text { with } \lim _{q \rightarrow 1+0} \chi(q)=1 .
$$


Given a number $1 \leq Q<2$, we choose $p$ and $q$ in such a way that

$$
q>1, \quad \chi(q)<\frac{2 q}{Q}, \quad \text { and } \quad \frac{1}{p}+\frac{1}{q}=1 .
$$

Theorem 6. Let $K>0,1 \leq Q<2, B \geq q Q \chi(p) / p(2 q-\chi(q) Q), \lambda_{0}>0$, and $T>0$ be given numbers, where $p$ and $q$ are defined in (4.10). Suppose that there exist a function $g(a, b)$ satisfying the conditions in (1.6) and a function $\phi(\lambda)$ defined for $\lambda>\lambda_{0}$ such that $\phi(\lambda)>0$. Suppose also that condition (4.9) is satisfied,

$$
E \exp \left(\lambda X_{a, b}\right) \leq K \exp (\phi(\lambda) g(a, b)) \text { for all } \lambda>\lambda_{0} \text { and } 0 \leq a<b \leq T,
$$

and

$$
M_{T}:=\sup _{\lambda>\lambda_{0}} \sup _{0 \leq a<b \leq T} \frac{E \exp \left(\lambda M_{a, b}\right)}{\exp (B \phi(\lambda) g(a, b))}<\infty .
$$

Then

$$
E \exp \left(\lambda M_{a, b}\right) \leq\left(1+K^{1 / p}\right)^{p} \exp (B \phi(\lambda) g(a, b))
$$

for all $\lambda>\lambda_{0}$ and $0 \leq a<b \leq T$.

It is easy to check that Theorem 5 is the particular case of Theorem 6 when $\phi(\lambda):=\lambda^{2}$.

\section{REFERENCES}

1. J. L. Doob, Stochastic processes, Wiley, New York, 1953.

2. L. Gerencsér, On a class of mixing processes, Stochastics 26 (1989), 165-191.

3. E. J. Hannan, The asymptotic theory of linear time-series models, J. Appl. Probab. 10 (1973), 130-145.

4. E. J. Hannan and M. Deistler, The statistical theory of linear systems, Wiley, New York, 1988.

5. F. Móricz, Moment inequalities and the strong laws of large numbers, $\mathrm{Z}$. Wahrsch. Verw. Gebiete 35 (1976), 299-314.

6. Sci. Math. (Szeged) 38 (1976), 325-341.

7. F. A. Móricz, R. J. Serfling, and W. F. Stout, Moment and probability bounds with quasisuperadditive structure for the maximum partial sum, Ann. Probab. 10 (1982), 1032-1040.

8. J. Rissanen, Stochastic complexity in statistical inquiry, World Sci. Publ., Singapore and Teaneck, NJ, 1989.

9. M. S. Taqqu, Self-similar processes and related ultraviolet and infrared catastrophes, Random Fields: Rigorous Results in Statistical Mechanics and Quantum Field Theory, North-Holland, Amsterdam, 1980.

BOLyAI INSTITUTE, UNIVERSITY OF SZEGEd, ARADI VERTANGK TERE 1, 6720 SZEGED, HUNGARY 